\title{
Gestational Trophoblastic Diseases: A Review of the Clinical Presentation and Management at the Alex Ekwueme Federal University Teaching Hospital Abakaliki
}

\author{
Nwafor Johnbosco Ifunanya*, Obi Vitus Okwuchukwu, Ibo Chukwunenye Chukwu, \\ Obi Chuka Nobert, Onwe Blessing, Ugoji Darlington-Peter Chibuzor, \\ Onuchukwu Victor Uchenna \\ Department of Obstetrics and Gynaecology, Alex Ekwueme Federal University Teaching Hospital, Abakaliki, \\ South-East Nigeria, Nigeria \\ Email: *nwaforjohnbosco97@gmail.com
}

How to cite this paper: Ifunanya, N.J., Okwuchukwu, O.V., Chukwu, I.C., Nobert, O.C., Blessing, O., Chibuzor, U.D.P. and Uchenna, O.V. (2019) Gestational Trophoblastic Diseases: A Review of the Clinical Presentation and Management at the Alex Ekwueme Federal University Teaching Hospital Abakaliki. Case Reports in Clinical Medicine, 8, 164-171.

https://doi.org/10.4236/crcm.2019.86020

Received: May 27, 2019

Accepted: June 21, 2019

Published: June 24, 2019

Copyright $\odot 2019$ by author(s) and Scientific Research Publishing Inc. This work is licensed under the Creative Commons Attribution International License (CC BY 4.0).

http://creativecommons.org/licenses/by/4.0/

\begin{abstract}
Background: Gestational trophoblastic diseases (GTD) are potentially curable with retention of reproductive function once the correct diagnosis is made and treatment is commenced early with adequate follow up. Objective: The objective of this study was to determine the incidence, clinical presentation, management and treatment outcomes of gestational trophoblastic diseases in a tertiary hospital in Abakaliki, South-east Nigeria. Materials and Methods: This was a retrospective descriptive study of gestational trophoblastic diseases managed at Alex Ekwueme Federal University Teaching Hospital, Abakaliki (AEFUTHA) over a 6-year period. The statistical analysis was done using SPSS version 22. Results: The incidence of GTD was 4.6 per 1000 deliveries. Women who were less than 20 years and more than 40 years of age accounted for $5.9 \%$ and $23.5 \%$ of cases of GTD respectively. Women who were para 5 and above accounted for $76.5 \%$ of those who presented with GTD. All the patients presented with vaginal bleeding. Suction evacuation (76.5\%) was the commonest form of treatment offered to women with GTD. The commonest complication was anaemia (94.1\%). Maternal death due to GTD was $8.8 \%$. Most (58.8\%) of the patients did not turn up for follow-up. Conclusion: Gestational trophoblastic diseases has remained an important cause of maternal morbidity and mortality in our hospital due to poor compliance with follow-up. Call and recall system should be introduced in the management of patients with GTD to improve compliance to management standard.
\end{abstract}




\section{Keywords}

Gestational, Maternal, Mortality, Chemotherapy, Follow-Up

\section{Introduction}

The history of the management of gestational trophoblastic diseases can be considered as one of the success stories of modern medicine, as the majority, if not all, are potentially curable with the retention of reproductive function once the correct diagnosis is made and treatment is commenced early [1]. Gestational trophoblastic disease defines a heterogeneous group of interrelated lesions arising from the trophoblastic epithelium. They include hydatidiform mole, invasive mole, placental site trophoblastic tumour and choriocarcinoma [1]-[6].

The incidence of GTD varies greatly throughout the world [1] [2] [3] [4]. The exact incidence in Nigeria is not known [4]. Gestational trophobastic tumours account for $5.3 \%$ of gynaecological tumours in a study done in Enugu [4]. In Kano, the incidence of GTD was 4.5 per 1000 deliveries [2].

The aetiology of GTD is not as well understood [4]. However, it has been associated with ethnicity, extremes of reproductive age ( $<20$ years and $>40$ years), prior molar pregnancy, lower socioeconomic class and dietary deficiency of protein, folic acid and carotene [1] [2] [3].

Gestational trophoblastic diseases are usually suspected in patients with amenorrhoea presenting with vaginal bleeding, the passage of grape-like vesicles, hyperemesis gravidarium, fundal height greater than estimated gestational age and positive serial hCG estimation [1]-[8].

Complications associated with GTD include haemorrhage, anaemia, preeclampsia prior to 20 weeks gestation, thyrotoxicosis, shock and death [3] [4] [5].

Ultrasound is a non-invasive, safe, economical and relatively simple technique used in the diagnosis of GTD [2] [4]. The definitive diagnosis is made by histological examination of the products of conception [4].

The treatment of GTD depends on the clinico-histopathological type and may include suction evacuation, chemotherapy, hysterectomy and radiotherapy [4].

Follow up is essential in all cases of GTD so that persistent active trophoblastic tissue will be detected [1]-[10]. Follow-up is by history, physical examination, serial quantitative B-hCG estimation and chest X-ray when indicated. Pregnancy is discouraged during follow-up to avoid interfering in patient's B-hCG monitoring, while an effective contraceptive method is commenced and maintained throughout the period of surveillance [3] [5] [6] [7].

The risk of recurrence is $1 \%$ [3]. A woman who has had 2 molar gestation has a recurrence rate of $15 \%-28 \%$ [3]. As a result, the patient should be advised to report to the hospital once she misses her period after follow-up [3].

Gestational trophoblastic diseases are of great interest because of their excellent prognosis if diagnosed and treated early and the potential for child bearing 
can be maintained [7].

Considering the poor outcome in our patients of this otherwise generally good prognostic condition, the study is important. The findings of this study may help the policy makers and other stakeholders plan on how to implement appropriate strategies to reduce the maternal morbidity and mortality arising from it.

The objective of this study was to determine the incidence, clinical presentation, management and treatment outcomes of gestational trophoblastic diseases.

\section{Materials and Method}

\subsection{Study Area}

Abakaliki is the capital of Ebonyi state located in the south-east geographical zone of Nigeria. It has a total population of 267,386 [11]. Alex Ekwueme Federal University Teaching Hospital is a tertiary institution located in Abakaliki. It receives referral from all parts of the state and neighbouring states of Benue, Enugu, Cross River and Abia as well as any part of the country. The Obstetrics and Gynaecology department has 5 teams. Each team is headed by the most senior consultant with the responsibility of training of resident doctors and house officers. The gynaecologic patients are seen at the Gynaecologic clinic and ward, Accident and Emergency department, Intensive care unit and theatre.

\subsection{Study Design}

This was a retrospective descriptive study of women who were managed for gestational trophoblastic diseases in the Federal Teaching Hospital, Abakaliki. The period under review was $1^{\text {st }}$ January 2012 to $31^{\text {st }}$ December 2017. The hospital numbers of women that had GTD during the study period were retrieved from the admission register in the gynaecological ward and intensive care unit. Then the case notes were retrieved from the Medical Records Department of the hospital using the hospital numbers. A proforma containing information on sociodemographic characteristics, risk factors such as age, parity and history of previous GTD, clinical presentations, complications, mode of treatment and follow up of patients was used to extract information from the case notes. The statistical analysis was done using Statistical Package for Social Sciences (SPSS) version 22 software.

\subsection{Ethical Consideration}

The approval for the study was obtained from the Research and Ethical Committee of the Alex Ekwueme Federal University Teaching Hospital, Abakaliki.

\section{Results}

There were 68 cases of gestational trophoblastic. During the study period, there were 14,636 deliveries and 3100 gynaecological admissions. The incidence of GTD was 4.6 cases per 1000 deliveries and it accounted for $2.2 \%$ of gynaecological admission. 
The mean age at diagnosis was $32.1 \pm 4.6$ years. Women who were less than 20 years and more than 40 years of age accounted for $5.9 \%$ and $23.5 \%$ of cases of GTD respectively (Table 1). Majority of women (61.8\%) were farmers and $44.1 \%$ had no formal education. Women who were para 5 and above accounted for $76.5 \%$ of those who presented with GTD (Table 1). The commonest clinical presentation was vaginal bleeding (100\%) while $97.1 \%$ of women had amenorrhoea. Only $2.9 \%$ of patients had previous history of GTD (Table 2). The commonest complication was anaemia (94.1\%). Acute haemorrhage accounted for $70.6 \%$ of complications of GTD in the patients. Three (8.8\%) of the patients that were managed for metastatic choriocarcinoma suffered maternal death during follow up (Table 2).

Suction evacuation (76.5\%) was the commonest form of treatment offered to women with hydatidiform mole in FETHA (Table 3). Four (5.9\%) patients with hydatidiform mole were offered hysterectomy for uncontrollable haemorrhage following suction evacuation. Sixteen (23.5\%) patients had chemotherapy in cases of choriocarcinoma while twelve women with hydatidiform mole had chemotherapy using methotrexate in cases of persistent rise or plateau of B-hCG. For all cases of choriocarcinoma, WHO scoring was done. Three cases of low risk patient (score $\leq 6)$ received single agent chemotherapy with methotrexate while five cases of high risk patient received chemotherapy with etoposide, methotrexate, actinomycin D, cyclophosphamide and vincristine [EMACO] regimen.

Table 1. Sociodemographic characteristics of women with GTD.

\begin{tabular}{ccc}
\hline Characteristics & Frequency & Percentage \\
\hline Age (years) & & \\
$\leq 20$ & 4 & 5.9 \\
$21-39$ & 48 & 70.6 \\
$\geq 40$ & 16 & 23.5 \\
Occupation & & \\
Housewife & 4 & 5.8 \\
Farmer & 42 & 61.7 \\
Self employed & 18 & 5.8 \\
Civil servant & 4 & 26.4 \\
Educational status & & \\
No formal education & 30 & 44.1 \\
Primary & 24 & 35.2 \\
Secondary & 12 & 17.6 \\
Tertiary & 2 & 2.9 \\
Parity & & \\
0 & 2 & 2.9 \\
$1-4$ & 14 & 20.5 \\
$\geq 5$ & 56.4 \\
\hline
\end{tabular}


Table 2. Clinical presentation and complications of GTD.

\begin{tabular}{ccc}
\hline Features & Frequency & Percentage \\
\hline Clinical presentation & & \\
Amenorrhoea & 66 & \\
Vaginal bleeding & 68 & 97.1 \\
Previous history of GTD & 2 & 100.0 \\
Abdominal swelling & 46 & 2.9 \\
Excessive vomiting & 24 & 67.6 \\
Passage of vesicles & 32 & 35.2 \\
Fundal height $>$ gestational age & 64 & 47.1 \\
Cough & 6 & 94.1 \\
Complications & & 8.8 \\
Acute haemorrhage & 48 & \\
Anaemia & 64 & 70.5 \\
Preeclampsia/Hypertension & 8 & 94.1 \\
Thyrotoxicosis & 2 & 11.7 \\
Shock & 16 & 2.9 \\
Death & 6 & 23.5 \\
& & 8.8 \\
\hline
\end{tabular}

Table 3. Mode of treatment offered to women with GTD.

\begin{tabular}{ccc}
\hline Treatment method & Frequency & Percentage \\
\hline Suction evacuation only & 52 & 76.4 \\
Suction evacuation with chemotherapy & 12 & 17.6 \\
Suction evacuation with hysterectomy & 4 & 5.8 \\
Hysterectomy only & 0 & 0.0 \\
Hysterectomy with chemotherapy & 0 & 0.0 \\
Chemotherapy only & 16 & 23.5 \\
\hline
\end{tabular}

All the patients were offered contraception during treatment for GTD. Barrier methods using male condom was the commonest contraceptive method of choice for women with GTD. Twenty-two (36.7\%) patients used subdermal Levonorgestrel for contraception (Table 4).

Majority of the women (58.8\%) did not turn up for follow-up. Twenty patients had follow-up for 1 year (Table 5). During follow-up, patients were evaluated with the serum B-hCG levels, ultrasound, chest X-ray (if indicated) and menstrual cycle of patients were enquired.

There were sixteen $(23.5 \%)$ cases of choriocarcinoma and fifty-two $(76.5 \%)$ cases of hydatidiform mole. There was no case of invasive mole or placental site trophoblastic tumour (Table 6). 
Table 4. Contraceptive methods offered to women with GTD.

\begin{tabular}{ccc}
\hline Contraceptive method & Frequency & Percentage \\
\hline Male condom & 34 & 50.0 \\
Subdermal Levonorgestrel & 22 & 32.3 \\
Combined oral contraceptive pill & 12 & 17.6 \\
\hline
\end{tabular}

Table 5. Duration of follow-up.

\begin{tabular}{ccc}
\hline Duration & Frequency & Percentage \\
\hline 0 - 6 months & 16 & 23.5 \\
6 months - 1 year & 12 & 17.6 \\
$>$ 1 year & 0 & 0.0 \\
Did not turn up for follow-up & 40 & 58.8 \\
\hline
\end{tabular}

Table 6. Histological type of GTD.

\begin{tabular}{ccc}
\hline Histology type & Frequency & Percentage \\
\hline Hydatidiform mole & 36 & \\
Complete & 16 & 53.0 \\
Partial & 16 & 23.5 \\
Choriocarcinoma & 0 & 23.5 \\
Invasive mole & 0 & 0 \\
Placental site trophoblastic tumour & & 0 \\
\hline
\end{tabular}

\section{Discussion}

The incidence of GTD in this study was 4.6 cases per 1000 deliveries. This finding shows that the condition is common in our environment. The incidence of GTD in this study is comparable to the incidence of 4.7 per 1000 deliveries reported in Nnewi [4] and 4.5 per 1000 deliveries in Kano [2]. However, it is much higher than incidences of 1 per 1000, 1.3 per 1000, and 2 per 1000 pregnancies reported in the United States, the United Kingdom and Japan respectively [2]. This high incidence of GTD in our centre may be due to the status of the teaching hospital as a referral centre from various clinics both private and public.

The aetiology of GTD is unknown [2]-[6]. However, there are several risk factors which include extremes of maternal age $<20$ years and $>40$ years, prior history of molar gestation, low socioeconomic status and high parity [3] [4]. Maternal age has been found to influence the risk of GTD; the incidence being more in women under the age of 20 years and rising sharply after the age of 40 years. The findings from this study do not support this; it is however in conformity with studies done in Jos and Nnewi [7] [8]. Women less than 20 years and those more than 40 years of age accounted for $29.4 \%$. A large proportion of the 
patients (76.5\%) were of high parity $(\geq 5)$ and this conforms with a report from some authors where most patients were of high parity [7]. A history of Previous molar gestation has consistently been shown to influence the risk of GTD [2]. However, in this study, only $3 \%$ of patients had a prior history of GTD. This is similar to a study in Jos in which $8 \%$ of patients had a previous history of GTD [8].

Vaginal bleeding and amenorrhoea are the most common clinical presentation of GTD in this study. It is, therefore, necessary for all patients with such complaints in early pregnancy to have ultrasound scan which is reliable in the diagnosis of the condition. Other common clinical features include abdominal swelling, the passage of vesicles and symphysiofundal height greater than gestational age. These findings are similar to the findings from similar studies [9].

Suction evacuation (76.5\%) was the preferred method of treatment for women with hydatidiform mole in this study. Suction evacuation allows for rapid evacuation of the uterus, reduces the danger of uterine perforation and provides specimen for histology. Only 5.9\% of patients had hysterectomy for uncontrollable haemorrhage following the suction evacuation. Chemotherapy was given to $23.5 \%$ of the patients. Chemotherapy was introduced in cases of persistent rise or plateau of B-hCG and in cases of choriocarcinoma. Studies have shown that the incidence of persistent GTD may be decreased with prophylactic chemotherapy. [9] It is controversial whether prophylactic chemotherapy following evacuation should be offered to patients considered high risk for persistent gestational trophoblastic disease or whom poor compliance to follow up is anticipated [10]. However, prophylactic chemotherapy can be a viable option in our setting because of poor compliance to follow-up of our patients.

Follow up of patients with GTD is very important and it allows early detection of persistence disease [12]. Unfortunately, most (58.8\%) of the patients did not turn up for follow-up. The poor compliance to follow-up may be as a result of poverty, illiteracy, cultural attitudes and inadequate health education. Adequate counselling and introduction of call and recall system may help to improve compliance to treatment follow-up.

The mortality rate in this study is $8.8 \%$. This is comparable to $13.3 \%$ and $6.8 \%$ reported in Nnewi and Kano respectively [2] [4]. This was considerably high when compared to reports from developed nations [12]. The early diagnosis, adequate treatment and adherence to follow-up in developed nations could explain the documented good prognosis for this condition.

\section{Conclusion}

Gestational trophoblastic diseases have remained an important cause of maternal morbidity and mortality in our hospital. There is a need for early diagnosis and adequate treatment of this condition. Adequate follow-up of the patients should be re-emphasized. Call and recall system should be introduced in the management of patients with GTD to improve compliance to management standard. 


\section{Limitation}

The limitations of this study include the fact that there was poor compliance to follow-up such that the incidence of subsequent pregnancies after complete treatment of GTD was not studied.

\section{Conflicts of Interest}

The authors declare no conflicts of interest regarding the publication of this paper.

\section{References}

[1] Anfinan, N., Sait, K. and Sait, H. (2014) Gestational Trophoblastic Disease in the Western Region of Saudi Arabia (Single Institute Experience). European Journal of Obstetrics Gynecology and Reproductive Biology, 180, 8-11. https://doi.org/10.1016/j.ejogrb.2014.06.005

[2] Yakasai, I., Abubakar, I. and Eze, Y. (2015) Gestational Trophoblastic Diseases in a Teaching Hospital in Northern, Nigeria. American Journal of BioScience, 3, 7-10. https://doi.org/10.11648/j.ajbio.20150301.12

[3] Ghimire, S. (2012) Gestational Trophoblastic Disease: Experience at Nobel Medical College. Postgraduate Medical Journal of NAMS, 12, 9-12.

[4] Ozumba, B.C., Nzegwu, M.A. and Anyikam, A. (2011) Histological Patterns of Gynaecological Lesions in Enugu, Nigeria. A Five-Year Review from January 1, 2000 to December 31, 2004. Advances in Bioresearch, 2, 132-136.

[5] Joneborg, U., Eloranta, S., Johansson, A.L., Marions, L., Weiboll, C.E. and Lambe, M. (2014) Hydatidiform Mole and Subsequent Pregnancy Outcome: A Population-Based Cohort Study. American Journal of Obstetrics Gynecology, 211, 68-72. https://doi.org/10.1016/j.ajog.2014.06.030

[6] Shrivastava, S., Kataki, A.C., Barmon, D., Deka, P., Bhuyan, C. and Bhargav, S.J. (2014) Gestational Trophoblastic Neoplasia: A 6 Year Retrospective Study. South Asian Journal of Cancer, 3, 33-37. https://doi.org/10.4103/2278-330X.126516

[7] Ocheke, A.N., Musa, J. and Uamai, A.O. (2011) Hydatidiform Mole in Jos, Nigeria. Nigerian Medical Journal, 54, 223-226. https://doi.org/10.4103/0300-1652.93792

[8] Igwegbe, A.O. and Eleje, G.U. (2013) Hydatidiform Mole: A Review of Management Outcomes in a Tertiary Hospital in South-East Nigeria. Annals of Medical \& Health Sciences Research, 3, 210-214. https://doi.org/10.4103/2141-9248.113664

[9] Nnadi, C.D., Ango, I.G. and Nwobodo, E.I. (2015) Choriocarcinoma with Cerebral Metastasis Presenting as a Stroke-Like Lesion. Sahel Medical Journal, 18, 16-19. https://doi.org/10.4103/1118-8561.149498

[10] Almasi, A., Almassinokiani, F. and Akbari, P. (2014) Frequency of Molar Pregnancies in Health Care Centres of Tehran, Iran. Journal of Reproduction Infertility, 15, 157-160.

[11] Population of Abakaliki (2018). https://www.townsoftheworld.com/Nigeria/Ebonyi/Abakaliki/Information

[12] Nirmala, C.K., Azlin, M.I., Harry, S.R., Lim, P.S., Shafiee, M.N., Azurah, A.G., et al. (2013) Outcome of Molar Pregnancies in Malaysia: A Tertiary Centre Experience. Journal of Obstetrics and Gynaecology, 33, 191-193. https://doi.org/10.3109/01443615.2012.741150 